\title{
Dislocation density and Burgers vector population in fiber-textured Ni thin films determined by high-resolution X-ray line profile analysis
}

\author{
Csiszár, Gábor; Pantleon, Karen; Alimadadi, Hossein; Ribárik, Gábor; Ungár, Tamás
}

Published in:

Journal of Applied Crystallography

Link to article, DOI:

$10.1107 /$ S0021889811053234

Publication date:

2012

Document Version

Publisher's PDF, also known as Version of record

Link back to DTU Orbit

Citation (APA):

Csiszár, G., Pantleon, K., Alimadadi, H., Ribárik, G., \& Ungár, T. (2012). Dislocation density and Burgers vector population in fiber-textured $\mathrm{Ni}$ thin films determined by high-resolution X-ray line profile analysis. Journal of Applied Crystallography, 45(1), 61-70. https://doi.org/10.1107/S0021889811053234

\section{General rights}

Copyright and moral rights for the publications made accessible in the public portal are retained by the authors and/or other copyright owners and it is a condition of accessing publications that users recognise and abide by the legal requirements associated with these rights.

- Users may download and print one copy of any publication from the public portal for the purpose of private study or research.

- You may not further distribute the material or use it for any profit-making activity or commercial gain

- You may freely distribute the URL identifying the publication in the public portal 
Journal of

\section{Applied Crystallography}

ISSN 0021-8898

Received 19 September 2011 Accepted 10 December 2011

(C) 2012 International Union of Crystallography Printed in Singapore - all rights reserved

\section{Dislocation density and Burgers vector population in fiber-textured $\mathrm{Ni}$ thin films determined by high- resolution $\mathrm{X}$-ray line profile analysis}

\author{
Gábor Csiszár, ${ }^{a}$ Karen Pantleon, ${ }^{\mathrm{b}}$ Hossein Alimadadi, ${ }^{\mathrm{b}}$ Gábor Ribárik $^{\mathrm{a}}$ and Tamás \\ Ungár ${ }^{a *}$

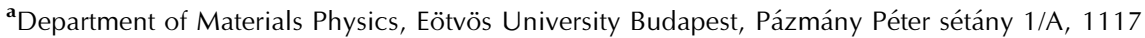 \\ Budapest, Hungary, and ${ }^{\mathbf{b}}$ Technical University of Denmark, Department of Mechanical \\ Engineering, Kemitorvet Building 425, DK - 2800 Lyngby, Denmark. Correspondence e-mail: \\ ungar@ludens.elte.hu
}

\begin{abstract}
Nanocrystalline Ni thin films have been produced by direct current electrodeposition with different additives and current density in order to obtain $\langle 100\rangle$, $\langle 111\rangle$ and $\langle 211\rangle$ major fiber textures. The dislocation density, the Burgers vector population and the coherently scattering domain size distribution are determined by high-resolution X-ray diffraction line profile analysis. The substructure parameters are correlated with the strength of the films by using the combined Taylor and Hall-Petch relations. The convolutional multiple whole profile method is used to obtain the substructure parameters in the different coexisting texture components. A strong variation of the dislocation density is observed as a function of the deposition conditions.
\end{abstract}

\section{Introduction}

During recent years, interest in nanocrystalline materials has increased enormously, because they provide superior multifunctional properties for advanced applications such as thin films, nanopowders or compact bulk nanocrystalline solids. In particular electrochemical deposition has become very attractive for dedicated fabrication of materials with microstructural features on the nanoscale (Natter \& Hempelmann, 2008; Moffat \& Jossell, 2010; Lu et al., 2009), because (i) the method is very cost efficient, (ii) it can be applied for the deposition of a huge number of different metals and alloys of complicated geometry, (iii) it allows great flexibility in tailoring the internal structure of the deposited materials, and (iv) it is well established in industry for large-scale production.

The internal structure and associated properties of electrochemically deposited films can be closely controlled by the applied process parameters, such as the chemical composition of the electrolytic bath, the type and level of the current density at the cathode (substrate), and the characteristics of the applied substrate material (e.g. Natter \& Hempelmann, 2008; Weil, 1989). Although the process parameters influence the as-deposited microstructure and properties of the films, the reliability and functionality of electrodeposited components is further affected by post-deposition microstructure changes occurring during self-annealing at room temperature and/or intentional annealing at elevated temperatures (Pantleon \& Somers, 2006, 2010; Hansen \& Pantleon, 2008). Traditional processing has been mainly targeted to tailor, in particular, the grain size in the as-deposited and the (self-) annealed state by means of appropriate selection of the deposition parameters or post-deposition treatments. For example, super-conformal film growth, as required for multilevel copper metallization in microelectronics (Moffat \& Jossell, 2010; Andricacos et al., 1998), relies on as-deposited nanocrystalline grains, but the electrical properties of the copper films require large defect-free grains as formed by subsequent (self-) annealing. For electrodeposited nickel and nickel-based alloys, the size of the grains has also been the main focus for tailoring the mechanical properties for the films' applications as microelectromechanical systems or as tools for electroforming (Erb, 2010).

However, although the traditional approach consists of controlling solely the size of grains for tailoring the properties of deposited materials, the lattice defects within the grains, and hence their substructure consisting of twins and dislocations, are also essential and allow unique combinations of properties, as reported recently for the example of electrodeposited copper films (Lu et al., 2009).

Quantification of the internal structure of materials on the nanoscale, including the substructure of grains, is not straightforward. Direct observation of the nanostructured thin films is limited, because of the resolution limit of (electron) microscopic analysis and/or the need for sample preparation, which carries with it the risk of introducing artefacts, in particular, in the non-equilibrium as-deposited films. X-ray diffraction (XRD), on the other hand, provides an indirect method for the characterization of the microstructure by meaningful interpretation of diffraction patterns. Although it does not allow direct imaging of the internal structure and provides absorption-weighted averages over the information depth of the X-rays rather than local microstructure information, appropriate XRD measurements can reveal not only 


\section{Table 1}

Crystallographic texture of nickel films, deposited at various current densities from a Watts bath containing different levels of the additive 2-butyne-1,4-diol (Alimadadi et al., 2012).

The fiber axis of the fiber texture is given as $\langle u v w\rangle$. The strengths of the fiber textures are characterized by the m.r.d. values given after each $\langle u v w\rangle$ direction.

\begin{tabular}{llll}
\hline & \multicolumn{2}{l}{ Current density } \\
\cline { 2 - 4 } $\begin{array}{l}\text { 2-Butyne-1,4-diol } \\
\text { concentration }\end{array}$ & $2 \mathrm{~A} \mathrm{dm}^{-2}$ & $5 \mathrm{~A} \mathrm{dm}^{-2}$ & $10 \mathrm{~A} \mathrm{dm}^{-2}$ \\
\hline $10 \mathrm{mMol} \mathrm{dm}^{-3}$ & $\langle 111\rangle 5.5$ & $\langle 111\rangle 2.5+\langle 511\rangle 1.6$ & $\langle 111\rangle 3.2+\langle 511\rangle 2.0$ \\
$5 \mathrm{mMol} \mathrm{dm}^{-3}$ & $\langle 211\rangle 2.7$ & $\langle 111\rangle 2.7+\langle 100\rangle 2.1$ & $\langle 100\rangle 32$ \\
$0 \mathrm{mMol} \mathrm{dm}^{-3}$ & $\langle 211\rangle 4.9$ & $\langle 100\rangle 5.5$ & $\langle 100\rangle 10$ \\
\hline
\end{tabular}

the size of grains but also details of the substructure within the grains (dislocation density and character, twin boundaries).

Using electrodeposited nickel films as an example, the present work reports a novel XRD analysis for evaluating nanostructured materials.

\section{Experimental}

\subsection{Thin-film deposition}

Nickel films were deposited onto an amorphous substrate applying electrochemical deposition from a Watts bath with different amounts of the organic additive 2-butyne-1,4-diol and varying current densities; for details on electrodeposition the reader is referred to Alimadadi et al. (2012). The thin amorphous substrate layer was deposited onto a copper sheet prior to the deposition of the nickel films. The copper sheet provided the strength of the substrate and the thin amorphous layer assured that the texture of the Ni films did not depend on the texture of the copper sheet but only on the circumstances of the deposition procedure. Different crystallographic preferred orientations of the $\mathrm{Ni}$ films were attained by varying the parameters in the deposition process. For all films, quantitative crystallographic texture analysis was carried out by applying conventional X-ray diffraction (D8 Discover diffractometer, Bruker AXS). The rotational symmetry of the measured pole figures clearly indicates the presence of ideal fiber textures; an example is shown in Fig. 1. The orientation

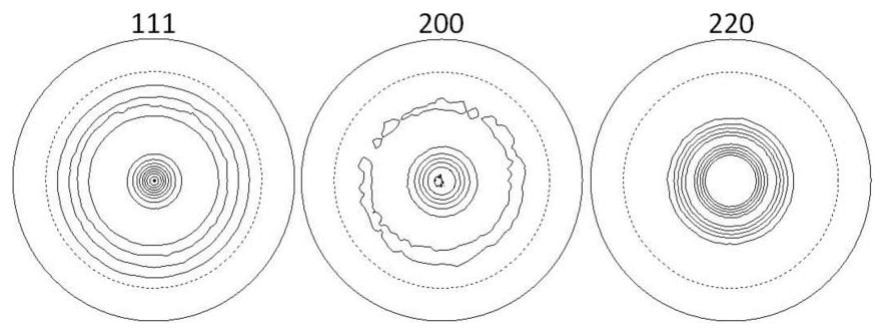

Figure 1

Pole figures measured for the 111, 200 and 220 reflections of a nickel film deposited at $2 \mathrm{~A} \mathrm{dm}^{-2}$ with an additive concentration of $5 \mathrm{mMol} \mathrm{dm}^{-3}$. The iso-intensity lines in the contour plots correspond to pole density levels drawn from 1 to 3 in steps of 0.25 . As for all other films, the rotational symmetry in the pole figures indicates the presence of ideal fiber textures. distribution function was calculated from the measured pole figures. The type and strength of the corresponding fiber axis were quantified from the inverse pole figures in the normal direction, i.e. in the direction of film growth in terms of multiples of random orientation distribution (m.r.d.). The different Ni films consist of either $\langle 111\rangle$ or $\langle 100\rangle$ or $\langle 211\rangle$ fiber textures or double fiber textures containing a main $\langle 111\rangle$ and an additional minor texture component as indicated in Table 1. The m.r.d. values for the different samples are also given in Table 1 . The thickness of the films amounts to about $15 \mu \mathrm{m}$.

\subsection{X-ray diffraction experiments}

The X-ray diffraction measurements were carried out using a high-resolution diffractometer with negligible instrumental effects and a fine-focus Co tube operated at $30 \mathrm{kV}$ and $30 \mathrm{~mA}$. The symmetrical 220 reflection of a plane Ge monochromator was used in order to eliminate the $K \alpha_{2}$ component and provide wavelength compensation at the position of the detector. The cross section of the beam impinging on the specimen was about $0.1 \times 1.5 \mathrm{~mm}$. The scattered radiation was registered by imaging plates (IPs) placed at a radius of $193 \mathrm{~mm}$ from the specimen. Typical IP images of the 111 and 311 reflections for one of the specimens are shown in Fig. 2. The diffraction patterns were obtained by integrating the intensity distributions along the corresponding Debye-Scherrer arcs. The height of the beam on the specimen surface was set to be $1.5 \mathrm{~mm}$, the distance from the specimen to the IP was $200 \mathrm{~mm}$ and the vertical divergence of the beam was about $4 \times 10^{-3}$. With these values the instrumental broadening of the peaks obtained from the central $25 \mathrm{~mm}$ of the images is less than $5 \times$ $10^{-4} \mathrm{~nm}^{-1}$, which is less than $5 \%$ in the case of the arcs with the strongest curvature. This geometrical error becomes exactly zero when the arcs straighten. Since line profile analysis depends neither on the absolute nor on the relative intensities of diffraction peaks, all Debye-Scherrer arcs are integrated within the same rectangular region in all the IPs used.
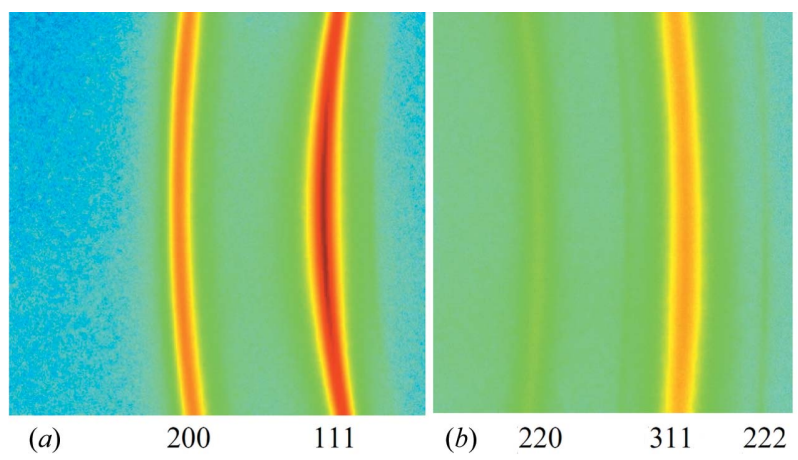

Figure 2

Typical IP images of the $111(a)$ and $311(b)$ reflections. The other, not labeled, reflections stem from grains not corresponding to the major $\langle 111\rangle$ texture. The image of the 111 reflection, elongated along the DebyeScherrer ring, indicates that the texture of the specimen is well defined but not sharp. 
Table 2

The angles, $\alpha$, between the incoming beam and the specimen surface satisfying the strict condition that each reflection corresponds to the $h \mathrm{kl}$ planes in the major texture component.

The $2 \theta$ values of the different reflections are also given in $(a)$ and $(b)$.

(a) $\langle 111\rangle$ texture.

\begin{tabular}{lllll}
\hline & 111 & 220 & 311 & 222 \\
\hline$\alpha$ & 26 & 10.6 & 27 & 61.5 \\
$2 \theta(\lambda=0.179 \mathrm{~nm})\left({ }^{\circ}\right)$ & 52.26 & 91.97 & 114.98 & 123.47 \\
\hline
\end{tabular}

(b) $\langle 100\rangle$ texture.

\begin{tabular}{lllll}
\hline & 200 & 220 & 311 & \multicolumn{2}{c}{222} \\
\hline$\alpha$ & 30.5 & $\sim 1$ & 32 & 7 \\
$2 \theta(\lambda=0.179 \mathrm{~nm})\left({ }^{\circ}\right)$ & 61.13 & 91.97 & 114.98 & 123.47 \\
\hline
\end{tabular}

(c) $\langle 211\rangle$ texture.

\begin{tabular}{lllll}
\hline & 111 & 220 & 311 & 222 \\
\hline$\alpha$ & 7 & 16 & 47 & 42 \\
\hline
\end{tabular}

In order to obtain diffraction always corresponding to the same fiber-texture component the angle between the incoming beam and the specimen surface, $\alpha$, has been selected in such a manner that the specific $h k l$ reflection corresponds to the $(h k l)$ planes of the selected texture component. This means that the four different reflections available in the reflection geometry for the specimens with different major fiber-texture components were measured by setting the values of the $\alpha$ angles for the different texture components and the different $h \mathrm{kl}$ reflections as listed in Table 2. The IP images of the 111 and 311 reflections shown in Figs. 2(a) and 2(b) for a $\langle 211\rangle$ fibertextured specimen are measured under asymmetric conditions with the tilt angles $\alpha=7^{\circ}$ and $\alpha=47^{\circ}$, respectively. 'Asymmetrical' diffraction conditions means here that the incoming and diffracted beams make different angles with the specimen surface. The 220 and 222 reflections indicated in Fig. 2(b) are still faintly visible in the IP. With the angle of the incident beam $\alpha=47^{\circ}$ these two reflections correspond to texture components other than the major one. The line profiles of such reflections will be used to evaluate the substructure in the nonmajor texture components of the specimen, as described in more detail below.

\section{Evaluation of the diffraction patterns}

The line profiles are evaluated by using the extended convolutional multiple whole profile (eCMWP) procedure (Ribárik et al., 2004; Balogh et al., 2006). The eCMWP software provides two options for the evaluation of diffraction peaks corresponding to specific texture components.

(i) The dislocation contrast factors for cubic crystals are coupled to each other by the $h k l$-dependent function for the average contrast factors,

$$
\bar{C}=\bar{C}_{h 00}\left(1-q H^{2}\right),
$$

where $q$ is a constant depending on the elastic anisotropy of the material and the character of dislocations, and $H^{2}=\left(h^{2} k^{2}+\right.$ $\left.h^{2} l^{2}+k^{2} l^{2}\right) /\left(h^{2}+k^{2}+l^{2}\right)^{2}$ (Ungár \& Tichy, 1999).

(ii) Individual contrast factors, $C_{\text {ind }}(h k l)$, can be attributed to each individual $h k l$ reflection. When using this second option a formal dislocation density, $\rho^{*}$, is selected as an input parameter for the evaluation, and the strain broadening of the profiles is obtained in terms of formal individual dislocation contrast factors, $C_{\text {ind }}^{*}(h k l)$. The mean-square strain, $\left\langle\varepsilon^{2}\right\rangle$, is proportional to the product of the contrast factors and the dislocation density, which also holds for the formal values: $\left\langle\varepsilon^{2}\right\rangle \simeq \rho C=\rho^{*} C^{*}$, where the products of the true and the formal values are equal, i.e. $\rho C=\rho^{*} C^{*}$ (Ungár \& Tichy, 1999). Once the values of $C_{\text {ind }}^{*}(h k l)$ are obtained the true dislocation density is provided by $\rho=\rho^{*} C^{*} / C$. The true values of the contrast factors can be determined theoretically, as shown in $\S 4$. It is noted that the $e C M W P$ software also offers the option to link the contrast factors corresponding to higher harmonics of $h \mathrm{kl}$ reflections, e.g. in the present case, when the measured 111 and 222 reflections correspond to the same texture component, the appropriate $C_{\text {ind }}^{*}(111)$ and $C_{\text {ind }}^{*}(222)$ are linked to be identical.

In the present work, a further option provided by the $e C M W P$ software is used for the simultaneous evaluation of the substructure in the different coexisting texture components.

(iii) In the $e C M W P$ software the diffraction patterns are evaluated by using reciprocal-space variables, i.e. $K=2 \sin \theta / \lambda$, where $\theta$ and $\lambda$ are the diffraction angle and the wavelength of $\mathrm{X}$-rays. The shape and broadening of each profile is only relevant around the center of each fundamental $h \mathrm{kl}$ peak. Keeping this in mind, the four different diffraction patterns measured at the four different angle settings of $\alpha$ listed in Table 2 are successively combined by shifting the corresponding $K$ scales appropriately. This technical procedure enables us to feed the four patterns into the $e C M W P$ software at the same time so that it can work on all the measured peaks simultaneously. It is important to note that the $h k l$ peak positions of the profiles are strictly used in the evaluation procedure as determined by the $h k l$ values and the lattice constant of the material. In this option the $e C M W P$ software allows the evaluation of peak profiles corresponding to different grain populations but to the same $d$ values, where $d=$ $\lambda / 2 \sin \theta$. Obviously, in this case the component of the peak broadening arising from the strain must be described in terms of individual dislocation contrast factors, $C_{\text {ind }}^{*}(h k l)$, as described in (ii) above.

The two options (ii) and (iii) are used for evaluating all four measured diffraction patterns simultaneously for the substructure in the major texture components (Ma), in the fraction of grains with random orientation $(\mathrm{R})$ and, if existing, in the minor texture components $(\mathrm{Mi})$ at the same time. Typical examples of the diffraction patterns obtained by tilting the specimen for the major texture components are shown in Figs. $3(a)-3(d)$. The artificial pattern for the purpose of feeding the data into the $e C M W P$ procedure is shown in Fig. 3(e) in logarithmic intensity scale. The patterns in Fig. 3 
were measured by orienting the sample for the $h k l$ reflections 200, 220, 311 and 222, respectively, corresponding always to the major texture component in line with the $\alpha$ angles given in Table 2. In these figures these reflections are denoted as $h k l$ Ma. The patterns are plotted versus $K=1 / d$ in reciprocal nanometre units. Technically the four patterns are fed into the $e C M W P$ software by creating an artificial pattern in which the patterns in Figs. $3(a)-3(d)$ are shifted in $K$ in order to obtain a two-column data file, where the first column contains the artificial $K$ values and the second the intensity distributions. The evaluation in the $e C M W P$ procedure is carried out by using the correct positions of the peaks in reciprocal space. Since the positions of the peaks in this figure are not absolute the scale is only given as a gauge.

The size component of the line broadening, related to the coherently scattering domains in the crystals, is also evaluated by the $e C M W P$ procedure. The $e C M W P$ method provides the size broadening in terms of the median and the logarithmic variance, $m$ and $\sigma$, for the lognormal size distribution function, $f(x)$ (Ribárik et al., 2004). A variety of average size values can be obtained as the different moments of the $f(x)$ function. Since transmission electron micrographs are the image of a thin planar section of the material, the size obtained from a transmission electron microscope image is closely correlated with the second moment of the $f(x)$ function, i.e. the area average crystallite size $\langle x\rangle_{\text {area }}=m \exp \left(2.5 \sigma^{2}\right)$ (Hinds, 1982). At the same time, these size values are closest to the subgrain size in transmission electron micrographs (Ungár et al., 2005).

\section{Evaluation of the dislocation structure}

\subsection{Dislocation densities in the different texture components}

Applying the $e C M W P$ procedure to the composite diffraction patterns, $C_{\text {ind }}^{*}(h k l)$ dislocation contrast factors are obtained for all diffraction profiles irrespective of which texture component they correspond to. These are the measured contrast factors. The theoretical contrast factors, $C_{\text {theor }}(h k l, \mathbf{b})$, pertaining to specific texture components were determined by using the $A N I Z C$ software, which calculates contrast factors for all conceivable combinations of the $h k l$ reflections and the Burgers vector, b, line vector, $\mathbf{l}$, and slip plane, $\mathbf{n}$, of dislocations, taking into account elastic anisotropy (Borbély et al., 2003). In the case of the reflections of the major texture components the values of $C_{\text {theor }}(h k l, \mathbf{b})$ were determined for 12 edge and six screw dislocations, each pertaining to a $\{111\}$ slip plane and [110] Burgers vector type. The $h k l$ subreflections were restricted to those that are contributing to diffraction at the particular texture component. For example, in the case of the $\langle 111\rangle$ textured specimen for the $\{220\}$ reflection only 220,202 and 022 , or in the case of the $\langle 100\rangle$-textured specimen for the same $\{220\}$ reflection only the $220,202,2 \overline{2} 0$ and $20 \overline{2}$ subreflections, were considered, in a

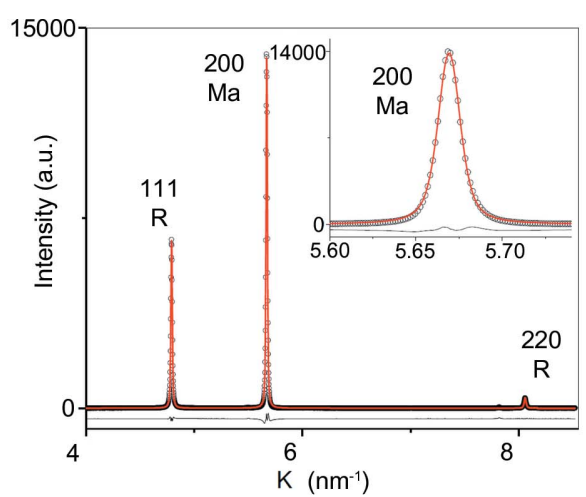

(a)

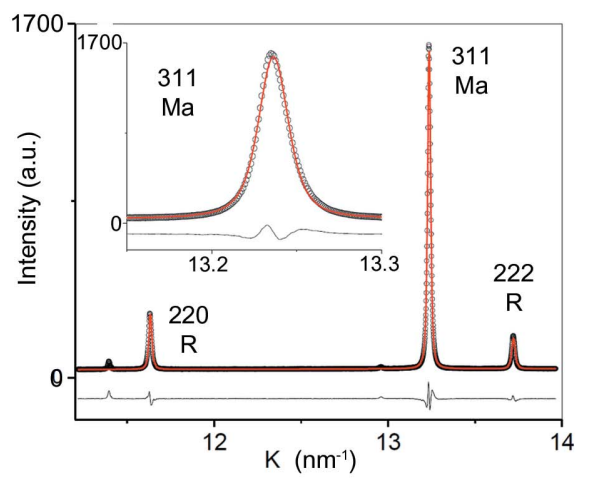

(c)

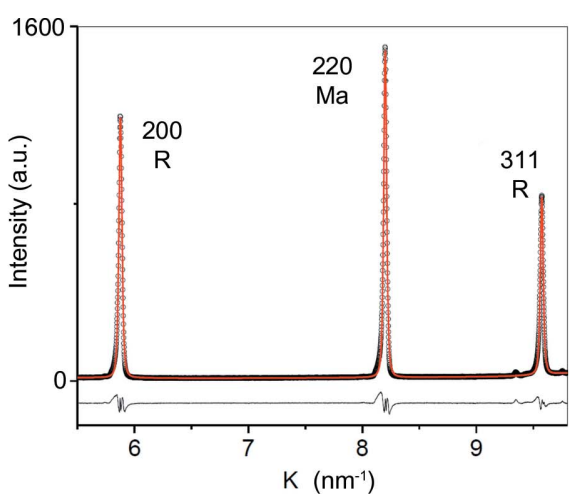

(b)

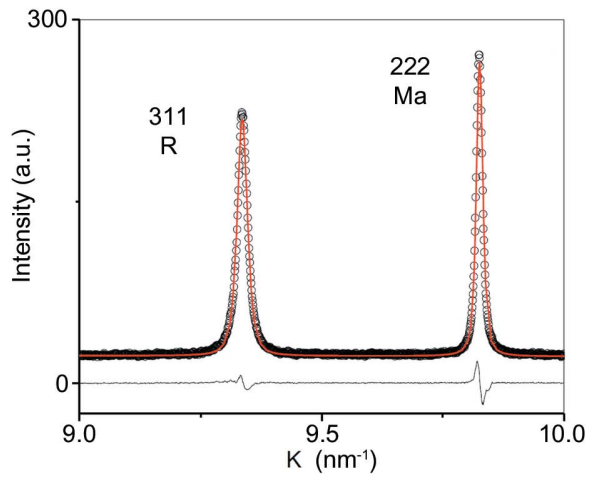

(d)

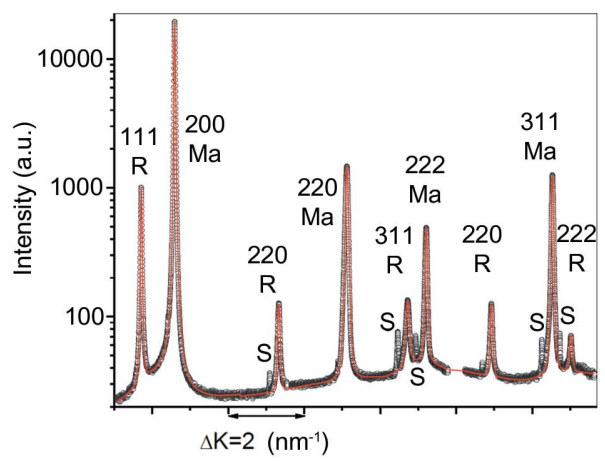

(e)

\section{Figure 3}

The measured (open circles) and the fitted [upper line (red in the electronic version of the journal); the lower grey line gives the difference] patterns of the $\langle 100\rangle$ fiber-textured specimen deposited with no additives and a current density of $10 \mathrm{~A} \mathrm{dm}^{-2} ;(a),(b),(c)$ and $(d)$ are the patterns measured by tilting the specimen for, respectively, the 200, 220, 311 and 222 reflections of the major (Ma) texture component into diffraction conditions. The other reflections, labeled as R, were evaluated for the randomly oriented crystallite population. (e) A typical artificial pattern for the purpose of feeding the data into the $e C M W P$ procedure. The horizontal scale can only be given here as a gauge; for more details see the text. The measured (open circles) and fitted (line) artificial patterns correspond to the $\langle 100\rangle$ fiber-textured specimen deposited with $5 \mathrm{mMol} \mathrm{dm}^{-3}$ additive and a current density of $10 \mathrm{~A}$ dm ${ }^{-2}$. Note the logarithmic intensity scale where the reflections coming through from the substrate (the copper sheet below the thin amorphous buffer layer) can also be seen and are labeled as ' $\mathrm{S}$ '. 
manner somewhat similar to that described by Cheary et al. (2000).

Minor texture components were only observed in the case of three specimens where, besides the $\langle 111\rangle$ major texture, $\langle 100\rangle$ or $\langle 511\rangle$ minority textures were also present. When possible, the dislocation densities in the minority texture components were also determined. Those reflections that did not correspond either to the major or to the minor texture components, and which appeared with considerably smaller intensities than those attributed to one of the texture components, were evaluated for dislocation densities in the nontextured randomly oriented fractions of the specimen.

\subsection{Burgers vector analysis in the major texture components}

Burgers vector analysis is carried out by matching the measured, $C_{\text {ind }}^{*}(h k l)$, and the theoretically calculated, $C_{\text {calc }}(h k l)$, dislocation contrast factors. We denote the theoretical contrast factors pertaining to the $i$ th subreflection and the $\mathbf{b}_{j}$ Burgers vector by $C_{\mathrm{th}}\left(h_{i} k_{i} l_{i}, \mathbf{b}_{j}\right)$. The theoretical contrast factor corresponding to the $h \mathrm{kl}$ reflection and Burgers vector $\mathbf{b}_{j}$ is

$$
\bar{C}_{\mathrm{th}}\left(h k l, \mathbf{b}_{j}\right)=\frac{1}{N^{*}} \sum_{i=1}^{N^{*}} C_{\mathrm{th}}\left(h_{i} k_{i} l_{i}, \mathbf{b}_{j}\right),
$$

where $N^{*} \leq N, N$ is the multiplicity of the $h k l$ reflection and $N^{*}$ is the number of selected $h k l$ reflections, where the selection takes into account the fiber texture of the foil. The strain-induced component of the line broadening is unanimously determined by the mean-square strain (Krivoglaz, 1969; Warren, 1959; Wilkens, 1970; Groma, 1998):

$$
\left\langle\varepsilon_{\mathrm{g}, L}^{2}\right\rangle=\frac{\rho C b^{2}}{4 \pi} f\left(L / R_{\mathrm{e}}\right)=\frac{\rho^{*} C_{\mathrm{ind}}^{*}(h k l) b^{2}}{4 \pi} f\left(L / R_{\mathrm{e}}\right),
$$

where $L$ is the Fourier variable, $R_{\mathrm{e}}$ is the effective outer cut-off radius of dislocations and $f\left(L / R_{\mathrm{e}}\right)$ is the Wilkens (1970) function. The calculated contrast factors conceivable for the different $h \mathrm{kl}$ reflections are calculated as the weighted sums of the theoretical contrast factors given in equation (1):

$$
\bar{C}_{\text {calc }}(h k l)=\frac{1}{J} \sum_{j} f_{j} \bar{C}_{\text {th }}\left(h k l, \mathbf{b}_{j}\right),
$$

where $j=1, \ldots, 18$ for the possible dislocations in facecentered cubic $\mathrm{Ni}, f_{j}$ is the weight of a particular dislocation and $J=\sum_{j} f_{j}$. The $f_{j}$ weights are determined by using a nonlinear least-squares Newton iteration procedure. In the present application the $e C M W P$ procedure starts by selecting a formal input value for the dislocation density, $\rho_{\text {input }}^{*}$, for which, as mentioned before

$$
\rho_{\text {input }}^{*} C_{\text {ind }}^{*}(h k l)=\rho \bar{C}_{\text {calc }}(h k l) .
$$

Since the $\rho_{\text {input }}^{*}$ value can be very different from the true dislocation density, the absolute values of the $C_{\text {ind }}^{*}(h k l)$ contrast factors can be very different from those of the $\bar{C}_{\text {calc }}(h k l)$ values. Therefore, the matching between the individual and calculated contrast factors has to be carried out by allowing for a scaling factor $A$ :
Table 3

Dislocation densities in the major texture components, $\rho_{\mathrm{Ma}}$, in the grains with random orientation, $\rho_{\mathrm{R}}$, and in the minor texture components, $\rho_{\mathrm{Mi}}$.

Specimen notation: S/additive concentration/deposition current/texture. The conditions of deposition are given in the first column. Numbers in parentheses are standard uncertainties and refer to the least significant digits.

\begin{tabular}{lllll}
\hline $\begin{array}{l}\text { Deposition } \\
\text { conditions } \\
\left(\mathrm{mMol} \mathrm{dm}^{-3},\right.\end{array}$ & $\begin{array}{l}\text { Specimen } \\
\text { notation }\end{array}$ & $\begin{array}{l}\rho_{\mathrm{Ma}} \\
\left(10^{15} \mathrm{~m}^{-2}\right)\end{array}$ & $\begin{array}{l}\rho_{\mathrm{R}} \\
\left(10^{15} \mathrm{~m}^{-2}\right)\end{array}$ & $\begin{array}{l}\rho_{\mathrm{Mi}} \\
\left(10^{15} \mathrm{~m}^{-2}\right)\end{array}$ \\
\hline 0,2 & $\mathrm{~S} / 0 / 2 /\langle 211\rangle$ & $1.2(2)$ & $0.5(1)$ & - \\
5,2 & $\mathrm{~S} / 5 / 2 /\langle 211\rangle$ & $6.0(10)$ & $6.1(10)$ & - \\
0,10 & $\mathrm{~S} / 0 / 10 /\langle 100\rangle$ & $1.3(2)$ & $0.9(2)$ & - \\
0,5 & $\mathrm{~S} / 0 / 5 /\langle 100\rangle$ & $2.0(2)$ & $0.7(1)$ & - \\
5,10 & $\mathrm{~S} / 5 / 10 /\langle 100\rangle$ & $9(2)$ & $9.1(20)$ & - \\
5,5 & $\mathrm{~S} / 5 / 5 /\langle 111\rangle+\langle 100\rangle$ & $4.2(10)$ & - & - \\
10,5 & $\mathrm{~S} / 10 / 5 /\langle 111\rangle+\langle 511\rangle$ & $4.5(10)$ & $5.8(10)$ & $14(3)$ \\
10,2 & $\mathrm{~S} / 10 / 2 /\langle 111\rangle$ & $4.4(10)$ & $5.9(10)$ & - \\
10,10 & $\mathrm{~S} / 10 / 10 /\langle 111\rangle+\langle 511\rangle$ & $23(4)$ & $12(2)$ & $26(5)$ \\
\hline
\end{tabular}

$$
\left[A \bar{C}_{\text {calc }}(h k l)-C_{\text {ind }}^{*}(h k l)\right]^{2}=\text { minimum, }
$$

where the scaling factor, $A$, provides the true dislocation density as

$$
\rho=A \rho_{\text {input }}^{*}
$$

The procedure described in equations (1)-(6) provides (i) the prevailing dislocation types, (ii) the prevailing Burgers vectors and (iii) the true dislocation densities.

\section{Results and discussion}

\subsection{Dislocation densities}

The dislocation densities vary in the different thin films between $1 \times 10^{15}$ and $23 \times 10^{15} \mathrm{~m}^{-2}$. The dislocation densities in the major, the random and the minor texture components, $\rho_{\mathrm{Ma}}, \rho_{\mathrm{R}}$ and $\rho_{\mathrm{Mi}}$, along with the error values, are given in Table 3. It can be seen that the $\rho_{\mathrm{R}}$ values are either equal to or smaller than the corresponding $\rho_{\text {Ma }}$ values. It is noted here that the error values in the tables are not the errors provided by the fitting procedure; rather they are the physical errors taking into account the reliability of the fits when changing the initial values.

Correlating the results of the substructure listed in Table 3 to the growth conditions of the films, given in Table 1, it is found that additive-free deposition causes the lowest dislocation densities in the range from $1 \times 10^{15}$ to $2 \times 10^{15} \mathrm{~m}^{-2}$, irrespective of the applied current density. With additive

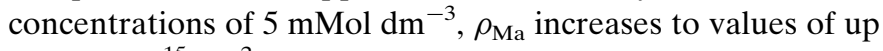
to $9 \times 10^{15} \mathrm{~m}^{-2}$ for the highest current density. With the largest concentration of the additive of $10 \mathrm{mMol} \mathrm{dm}^{-3}, \rho_{\mathrm{Ma}}$ increases further up to a value of $23 \times 10^{15} \mathrm{~m}^{-2}$. The dislocation density in the major texture components, $\rho_{\mathrm{Ma}}$, is plotted versus the concentration of the additive and the current density in Fig. 4. The figure indicates that, when the concentration of the additive is larger than about $5 \mathrm{mMol} \mathrm{dm}^{-3}$, the current density has a very strong effect on the increase of the dislocation density in the nickel films. 
The dislocation densities provided by the X-ray measurements are rather large, as can be seen in Table 3. In order to crosscheck these large $\rho$ values, the specimen with the largest concentration of the additive (i.e. $10 \mathrm{mMol} \mathrm{dm}^{-3}$ ) and the largest direct current density $\left(10 \mathrm{~A} \mathrm{dm}^{-2}\right)$ was annealed at $673 \mathrm{~K}$ for $30 \mathrm{~min}$. Sections of the as-deposited and annealed patterns with the 311 and 222 reflections are shown in Fig. 5. The figure shows the drastic narrowing of the peaks of the annealed sample. The pattern of the annealed specimen has been evaluated by the $e C M W P$ method and the dislocation density is obtained to be $0.16(5) \times 10^{15} \mathrm{~m}^{-2}$. In the asdeposited specimen this value is $\rho=23(2) \times 10^{15} \mathrm{~m}^{-2}$, indicating that after annealing the dislocation density drops by more than two orders of magnitude. It is worth noting that the FWHM values of the annealed specimen in the WilliamsonHall plot show qualitatively the same strain anisotropy as that of the as-deposited sample in Fig. 6(c) (open circles), which will be discussed later. The Burgers vector analysis for the

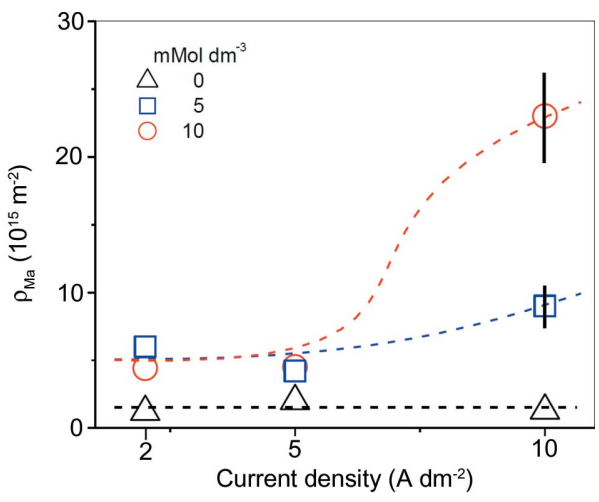

\section{Figure 4}

Correlation between one of the deposition conditions, namely direct current density, and the measured dislocation densities for all of the investigated samples with different major texture components. Classification is made by the different concentration of the additive solution. Significant changes as a function of the direct current density are observed in the dislocation densities for samples derived using $10 \mathrm{mMol} \mathrm{dm}^{-3}$ additive.

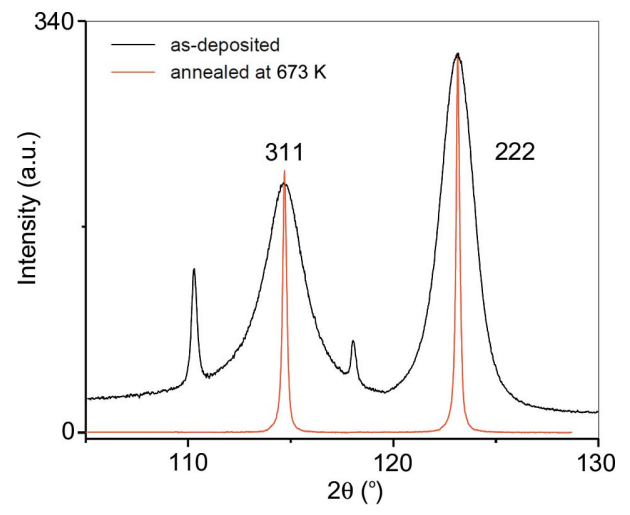

\section{Figure 5}

The 311 and 222 reflections of the as-deposited and annealed specimens with $10 \mathrm{mMol} \mathrm{dm}^{-3}$ additive concentration and $10 \mathrm{~A} \mathrm{dm}^{-2}$ current density as process parameters. The small peaks on the as-deposited sample profiles correspond to the substrate. The intensity ratios of the two peaks are in correlation with the 111 texture of the specimens. annealed specimen also reveals the same population as in the as-deposited sample.

\subsection{Burgers vector populations}

The qualitative features of line broadening are best shown in the Williamson-Hall (WH) plots of the FWHM scaled in $\mathrm{nm}^{-1}$, where FWHM $=(2 \cos \theta / \lambda) \Delta \theta$. Fig. 6 shows the WH plots of the different specimens with all three major textures. Strong strain anisotropy (Ungár, 2008) can be observed for all three textures; however, the qualitative behavior of this depends on the specific fiber-texture component. In the case of the $\langle 211\rangle$-textured specimens, in Fig. 6(a), the anisotropy is in good correlation with the assumption that the Burgers vector populations are random, e.g. the FWHM values of the 311

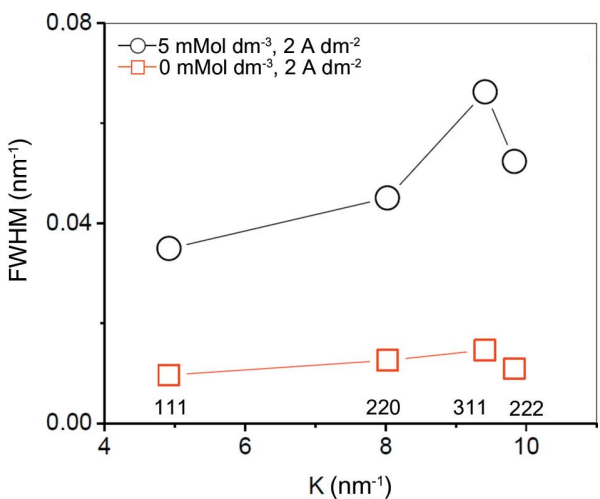

(a)

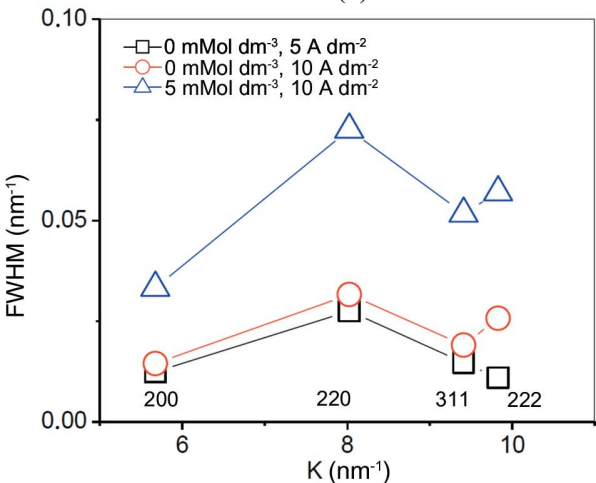

(b)

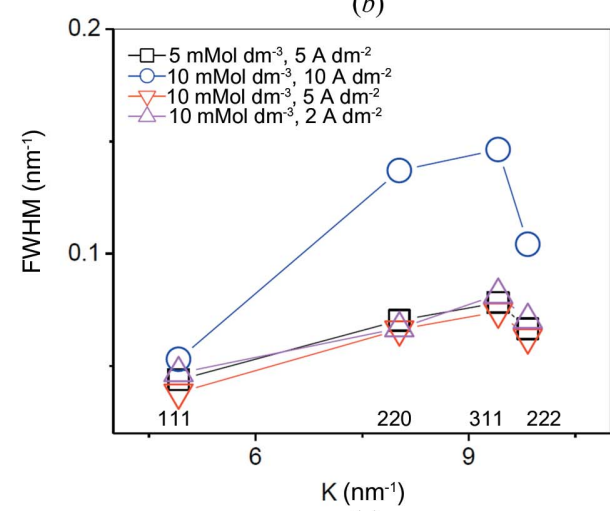

(c)

Figure 6

The Williamson-Hall plots of the $(a)\langle 211\rangle-,(b)\langle 100\rangle-$ and $(c)\langle 111\rangle-$ textured specimens. The additive concentration (in $\mathrm{mMol} \mathrm{dm}^{-3}$ ) and the current density (in $\mathrm{A} \mathrm{dm}^{-2}$ ) are given to identify the corresponding specimens in the legend of the figures. 
reflections are larger than the values of the 220 and 222 reflections. The Burgers vector analysis, as described in $\$ 4.2$, provides equal fractions for all possible Burgers vectors, within experimental error. Therefore, in these specimens the strain broadening has also been evaluated by assuming that the dislocation contrast factors are coupled by the relation given in equation (1).

In the case of the $\langle 100\rangle$ - and $\langle 111\rangle$-textured specimens, the WH plots in Figs. 6(b) and 6(c) show a qualitatively different strain anisotropy compared to that in Fig. $6(a)$ for $\langle 211\rangle$ textured films. In Fig. 6(b) the FWHM of the 220 reflection is considerably larger than that of 311, or in Fig. 6(c) the FWHM of 220 is almost equal to that of 311 , and is much larger than that of the 222 reflection. This behavior indicates that only specific Burgers vectors are present in the specimen, and therefore the contrast factors cannot be averaged, either over the $h k l$ permutations (because of the texture) or over the Burgers vector populations.

A note has to be added here about the anisotropy of the shape of crystallites in the films, in which, most often, columnar grains are observed. Taking into account the $\alpha$ tilts listed in Table 2, it can be shown that shape anisotropy cannot account for the anisotropic line broadening. For example, in the case of the $\langle 100\rangle$-textured specimen, the 222 reflection is measured with the tilt angle $\alpha=7^{\circ}$ at the diffraction angle $\theta=$ $61.57^{\circ}$. If the $h k l$ anisotropy in Fig. $6(b)$ were related to shape anisotropy and one assumed that the coherent domains are elongated parallel to the growth direction, because of the shallow incident beam, this reflection would have to correspond to the shortest distance in the domain. As a consequence, this 222 reflection would reveal the largest broadening. Fig. 6(b) shows the opposite, substantiating that shape anisotropy cannot explain the observed $h k l$ anisotropy in the WH plots. Similar arguments hold also for the $\langle 111\rangle$ texture specimens.

Strain anisotropy in the WH plots is rectified in the modified Williamson-Hall (mWH) plots (Ungár \& Tichy, 1999) by replacing the variable $K$ by $K C_{\text {calc }}^{1 / 2}$. In the case of the $\langle 211\rangle$ texture specimen the mWH plot in Fig. 7(a) is constructed by using the average contrast factors with $q=1.8$ for equal fractions of edge and screw character dislocations. The $\mathrm{mWH}$ plots of the $\langle 100\rangle$ - and $\langle 111\rangle$-texture specimens are shown in Figs. $7(b)$ and $7(c)$, where $C_{\text {calc }}$ are the calculated contrast factors corresponding to the Burgers vectors listed in Tables 4(a) and 4(b), respectively. Here we note that the $\mathrm{mWH}$ plots are used only to crosscheck qualitatively the correctness of the calculated contrast factors, $C_{\text {calc }}$. Since the actual functional behavior of the breadth values versus $K C_{\text {calc }}^{1 / 2}$ depends on the different parameters analyzed in detail in the $e C M W P$ whole-pattern evaluation procedure, the linear or quadratic nature of these curves is not interpreted or discussed further. Once the FWHM values follow a smooth curve versus $K C_{\text {calc }}^{1 / 2}$ the calculated contrast factors are considered as most likely appropriate.

The Burgers vector populations prevailing in the $\langle 100\rangle$ - and $\langle 111\rangle$-texture specimens are listed in Tables $4(a)$ and $4(b)$. Table 4 indicates that either all or the majority of the Burgers vectors in these specimens are perpendicular to the major texture direction. The Burgers vector population of dislocations in the $\langle 211\rangle$-texture specimens, as mentioned before, is random.

In thin films, the Burgers vectors of dislocations adjusting the lattice mismatch between the layer and the substrate are often parallel to the interface (Demkowicz \& Hoagland, 2008) and, similarly, the Burgers vectors in multilayer structures are usually parallel to the interfaces between the individual layers (Akasheh et al., 2007; Levay et al., 1999). Most of the Burgers vectors found for the $\langle 100\rangle$ - and $\langle 111\rangle$-textured specimens in this work are parallel to the interface. This would indicate that the dislocations were grown into the growing grains during the

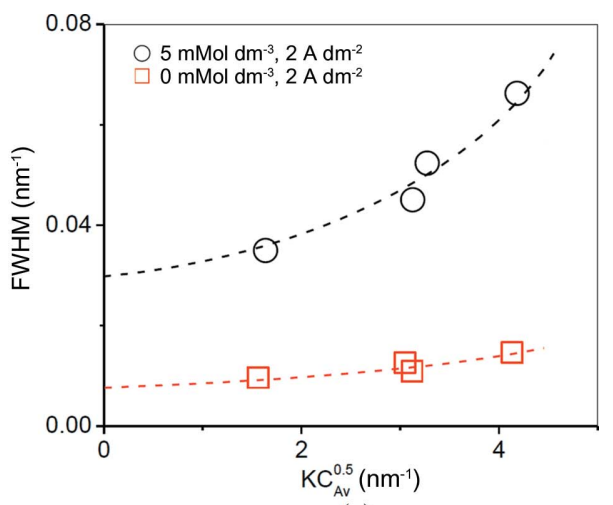

(a)

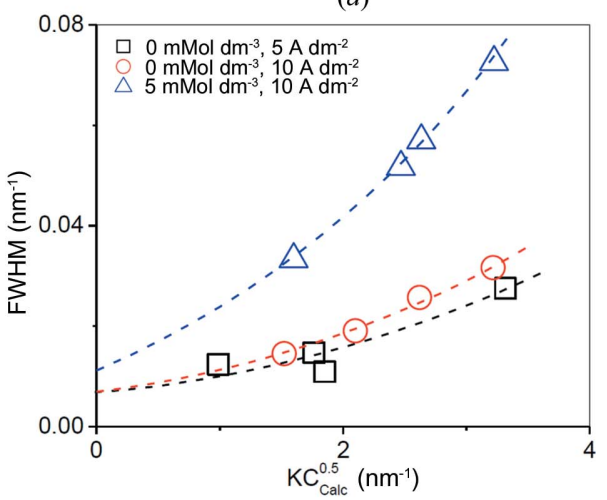

(b)

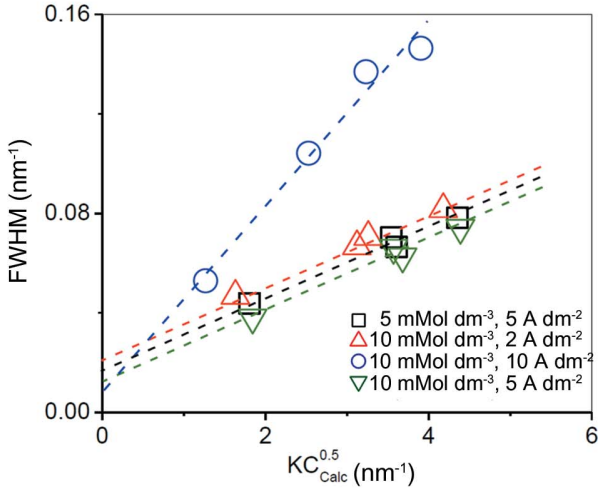

(c)

Figure 7

Modified Williamson-Hall plots of the same data as in Fig. 6: (a) $\langle 211\rangle$ texture, $(b)\langle 100\rangle$ texture and $(c)\langle 111\rangle$ texture. $C_{\mathrm{Av}}^{0.5}$ in $(a)$ indicates that here we used the average dislocation contrast factors. For more details see the text. 
electrolytic process. The in-plane dislocations reduce both the misfit between the substrate and the first growing layers of the film and, at later stages, the misfit between the atomic layers of the film. In the $\langle 211\rangle$-textured specimen the Burgers vectors turn out to be randomly oriented, suggesting that the face-centered cubic structure cannot be built up in a natural manner by the stacking of $\{211\}$-type planes. The evolution of this texture necessitates a threedimensional Burgers vector network in order to compensate for the misfits during the accession of the films. This indicates differences in the growth modes of the various electrodeposited films.

\subsection{The coherent domain size and the density of twin boundaries}

The size broadening was only determined for the peaks corresponding to the major texture component, and the $\langle x\rangle_{\text {area }}$ values are listed in Table 5. It can be seen that for the specimens with the same fiber-texture component the larger $\rho$ values correlate with smaller $\langle x\rangle_{\text {area }}$ values. It is observed that the area-weighted mean crystallite size is smallest, with values of between 20 and $40 \mathrm{~nm}$, in specimens with $\langle 111\rangle$ major texture components.

The $e C M W P$ procedure provides also the densities of twin boundaries, $\beta$, which are listed in Table 5 . The average distances between twin boundaries, $d_{\mathrm{twin}}$, are also given in the table, where $d_{\mathrm{twin}}=(100 /$ B) $d_{111}$ and $d_{111}$ is the distance of the 111 planes, $d_{111}=$ $0.204 \mathrm{~nm}$. The twin densities determined for all specimens with a major $\langle 111\rangle$ texture, except that deposited with the highest additive amount and the highest current density, are relatively large, well above the experimental error. For the other specimens the present method of X-ray line profile analysis did not reveal twin boundaries, although electron backscatter diffraction on the cross sections of the specimens clearly shows the presence of twinning (Alimadadi et al., 2012). The apparent discrepancy, however, can be understood in relation to the microscopically observed size of individual twins and the total volume fraction of twins with certain specific orientation: either (i) the distance of twin boundaries is beyond the limit of X-ray detectability, i.e. $d_{\mathrm{twin}} \geq 1 \mu \mathrm{m}$, or (ii) the total volume fraction of nanocrystalline twins of a certain orientation is too low for them to be detected by X-ray diffraction. In the case of (i) the line broadening caused by twinning is too small and in the case of (ii) the volume fraction of the, for example, $\langle 511\rangle$ twinned region is too small.

The coherent domain size given by the X-ray method is substantiated by plane-view transmission electron micrographs.

\subsection{The correlation between the strength and the substruc- ture of the thin foils}

For the purpose of correlating the dislocation densities with the strength of the foils, the Vickers hardness, HV, has been
Table 4

The Burgers vector populations prevailing in $(a)$ the $\langle 100\rangle$ - and $(b)$ the $\langle 111\rangle$ textured specimens.

$\mathbf{n}, \mathbf{b}$ and $\mathbf{I}$ indicates the slip plane, the Burgers vector and the line vector of the specific dislocations. The suffix $\mathrm{R}$ indicates a group of dislocations where the corresponding fraction numbers of the Burgers vector populations are the sum of the fractions for the dislocations that are identical to $\mathbf{b}_{\mathrm{R}}$ up to a rotational symmetry operation around the texture direction, i.e. for these dislocations at the given texture type the contrast factors are the same. The $\mathbf{n b l}$ values for all the random Burgers vectors, $\mathbf{b}_{\mathrm{R}}$, are $\langle 11 \overline{1}\rangle\langle 011\rangle\langle 2 \overline{1} 1\rangle$, $\langle 1 \overline{1} 1\rangle\langle 011\rangle\langle\overline{21} 1\rangle$ and $\langle 1 \overline{11}\rangle\langle 01 \overline{1}\rangle\langle 211\rangle$ for the $\langle 100\rangle$ fiber-textured specimen; and $\langle 11 \overline{1}\rangle\langle 011\rangle\langle 2 \overline{1} 1\rangle,\langle 1 \overline{1} 1\rangle\langle 011\rangle\langle\overline{21} 1\rangle,\langle 11 \overline{1}\rangle\langle 101\rangle\langle 1 \overline{21}\rangle,\langle\overline{11}\rangle\langle 101\rangle\langle\overline{12} 1\rangle,\langle 1 \overline{11}\rangle\langle 110\rangle\langle 1 \overline{1} 2\rangle$ and $\langle 1 \overline{1} 1\rangle\langle 110\rangle\langle\overline{1} 12\rangle$ for the $\langle 111\rangle$ fiber-textured specimen.

(a) $\langle 100\rangle$ fiber-textured specimen.

\begin{tabular}{|c|c|c|c|c|c|c|}
\hline \multicolumn{4}{|c|}{ Dislocation types } & \multicolumn{3}{|c|}{ Burgers vector population (\%) } \\
\hline & $\mathbf{n}$ & b & 1 & $\begin{array}{l}0 \mathrm{mMol} \mathrm{dm}^{-3} \\
10 \mathrm{~A} \mathrm{dm}^{-2}\end{array}$ & $\begin{array}{l}0 \mathrm{mMol} \mathrm{dm}^{-3} \\
5 \mathrm{~A} \mathrm{dm}^{-2}\end{array}$ & $\begin{array}{l}5 \mathrm{mMol} \mathrm{dm}^{-3}, \\
10 \mathrm{~A} \mathrm{dm}^{-2}\end{array}$ \\
\hline $\mathbf{b}_{\mathrm{R}}$ & $\begin{array}{l}11 \overline{1}_{\mathrm{R}} \\
- \\
-\end{array}$ & $\begin{array}{l}011_{\mathrm{R}} \\
011 \\
01 \overline{1}\end{array}$ & $\begin{array}{l}2 \overline{1} 1_{\mathrm{R}} \\
- \\
-\end{array}$ & $\begin{array}{l}60(20) \\
40(10) \\
-\end{array}$ & $\begin{array}{l}- \\
100(20) \\
-\end{array}$ & $\begin{array}{l}45(10) \\
30(10) \\
25(10)\end{array}$ \\
\hline
\end{tabular}

(b) $\langle 111\rangle$ fiber-textured specimen.

\begin{tabular}{|c|c|c|c|c|c|c|}
\hline \multicolumn{3}{|c|}{ Dislocation types } & \multicolumn{4}{|c|}{ Burgers vector population (\%) } \\
\hline $\mathrm{n}$ & b & $\mathbf{I}$ & $\begin{array}{l}5 \mathrm{mMol} \mathrm{dm}^{-3}, \\
5 \mathrm{~A} \mathrm{dm}^{-2}\end{array}$ & $\begin{array}{l}10 \mathrm{mMol} \mathrm{dm}^{-3} \text {, } \\
5 \mathrm{~A} \mathrm{dm}^{-2}\end{array}$ & $\begin{array}{l}10 \mathrm{mMol} \mathrm{dm}^{-3} \\
2 \mathrm{~A} \mathrm{dm}^{-2}\end{array}$ & $\begin{array}{l}10 \mathrm{mMol} \mathrm{dm}^{-3}, \\
10 \mathrm{~A} \mathrm{dm}^{-2}\end{array}$ \\
\hline $\mathbf{b}_{\mathrm{R}} 11 \overline{1}_{\mathrm{R}}$ & $011_{\mathrm{R}}$ & $2 \overline{1} 1_{R}$ & $40(10)$ & $40(10)$ & $40(10)$ & - \\
\hline 111 & $01 \overline{1}$ & $\overline{2} 11$ & $20(10)$ & $20(10)$ & $20(10)$ & $33(10)$ \\
\hline 111 & $10 \overline{1}$ & $\overline{1} 2 \overline{1}$ & $20(10)$ & $20(10)$ & $20(10)$ & $33(10)$ \\
\hline 111 & $1 \overline{1} 0$ & $11 \overline{2}$ & $20(10)$ & $20(10)$ & $20(10)$ & $33(10)$ \\
\hline
\end{tabular}

Table 5

The median, $m$, and the logarithmic variance, $\sigma$, of the lognormal size distribution function, the area average mean crystallite size, $\langle x\rangle_{\text {area }}=m \exp \left(2.5 \sigma^{2}\right)$, and the frequency of twin boundaries, $\beta$, for the specimens with different texture components, with the average distance of twin boundaries along the $\langle 111\rangle$ crystallographic direction, $d_{\mathrm{twin}}$, calculated from $\beta$.

Specimen notation: S/additive concentration/deposition current/texture. Numbers in parentheses are standard uncertainties and refer to the least significant digits.

\begin{tabular}{lrllll}
\hline Specimen notation & $m(\mathrm{~nm})$ & $\sigma$ & $\langle x\rangle_{\text {area }}(\mathrm{nm})$ & $\beta(\%)$ & $d_{\text {twin }}(\mathrm{nm})$ \\
\hline $\mathrm{S} / 0 / 2 /\langle 211\rangle$ & $70(10)$ & $0.30(5)$ & $90(20)$ & $0.00(2)$ & $>1 \mu \mathrm{m}$ \\
$\mathrm{S} / 5 / 2 /\langle 211\rangle$ & $30(5)$ & $0.10(2)$ & $30(10)$ & $0.0(2)$ & $>1 \mu \mathrm{m}$ \\
$\mathrm{S} / 0 / 10 /\langle 100\rangle$ & $45(5)$ & $0.71(10)$ & $160(25)$ & $0.00(2)$ & $>1 \mu \mathrm{m}$ \\
$\mathrm{S} / 0 / 5 /\langle 100\rangle$ & $55(5)$ & $0.30(5)$ & $70(15)$ & $0.00(2)$ & $>1 \mu \mathrm{m}$ \\
$\mathrm{S} / 5 / 10 /\langle 100\rangle$ & $55(5)$ & $0.15(2)$ & $60(15)$ & $0.00(2)$ & $>1 \mu \mathrm{m}$ \\
$\mathrm{S} / 5 / 5 /\langle 111\rangle+\langle 100\rangle$ & $6(2)$ & $0.71(10)$ & $22(5)$ & $0.54(10)$ & $37(7)$ \\
$\mathrm{S} / 10 / 5 /\langle 111\rangle+\langle 511\rangle$ & $10(3)$ & $0.71(10)$ & $35(7)$ & $3.1(3)$ & $6.5(10)$ \\
$\mathrm{S} / 10 / 2 /\langle 111\rangle$ & $29(5)$ & $0.32(5)$ & $37(7)$ & $3.8(3)$ & $5.3(10)$ \\
$\mathrm{S} / 10 / 10 /\langle 111\rangle+\langle 511\rangle$ & $6(2)$ & $0.71(10)$ & $22(5)$ & $0.00(2)$ & $>1 \mu \mathrm{m}$ \\
\hline
\end{tabular}

measured in a Zwick/Roell-ZH $\mu$-Indentec microhardness tester. The flow stress is obtained from the HV values as $\sigma=$ $\mathrm{HV} / 3$. We can assume that the dislocations, the grain size and the twins strengthen the foils by the Taylor and the Hall-Petch mechanisms at the same time, and that the flow stress can be written as (Taylor, 1934; Petch, 1953)

$$
\sigma=\sigma_{0}+\alpha \mu M_{\mathrm{T}} b \rho^{1 / 2}+K / d^{1 / 2},
$$

where $\sigma, \sigma_{0}, \alpha, \mu, b$ and $M_{\mathrm{T}}$ are the flow stress, the elastic limit, a constant between zero and unity, the shear modulus, the modulus of the Burgers vector, and the appropriate Taylor 


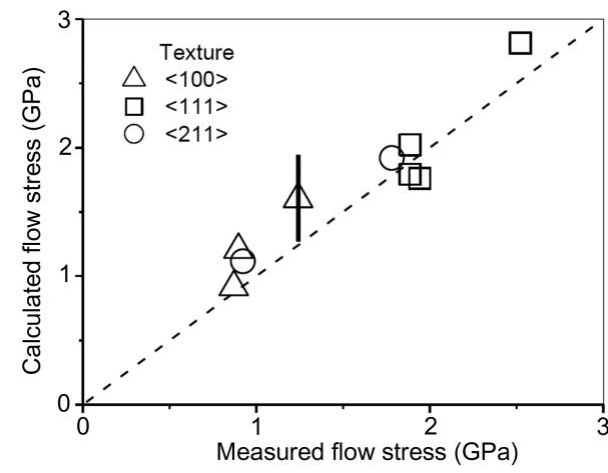

Figure 8

The calculated versus measured flow stress for the three different major textured classes.

factor, and $K$ is the Hall-Petch constant. The Taylor factors for the $\langle 100\rangle-,\langle 111\rangle$ - and $\langle 211\rangle$-texture specimens are taken as $M=$ 2.4, 3.2 and 3 (Clausen et al., 1998), respectively, where the m.r.d. is taken into account. The Hall-Petch constant for electrodeposited nanocrystalline $\mathrm{Ni}, \quad K=0.18 \mathrm{MPa} \mathrm{m}^{1 / 2}$ (Hansen, 2004), is used. For the value of $d$ the grain size, $\langle x\rangle_{\text {area }}$, as determined by the present X-ray method is taken. With $\sigma_{0}=220 \mathrm{MPa}, \mu=76 \mathrm{GPa}, b=0.2492 \mathrm{~nm}$ and $\alpha=0.15$ (2) the calculated and measured flow stress values are shown in Fig. 8. A good correlation between the measured and calculated flow stress values can be observed.

A final note of caution is needed. The method described and used here works better the stronger the texture of the specimen is. As the texture becomes weaker more and more grains not corresponding to the selected texture component contribute to a particular reflection measured at a specific sample orientation. These circumstances have to be taken into account in the interpretation of the experimental results and data.

\section{Conclusions}

(1) The convolutional multiple whole profile method of line profile analysis is extended to evaluate the substructure of coexisting texture components. The patterns are transformed from $I(2 \theta)$ to $I(K)$, where $K=2 \sin \theta / \lambda$ is the reciprocal-space coordinate. The $I(K)$ patterns, even when consisting of peaks corresponding to the same $h \mathrm{kl}$ indices but to different texture components, are evaluated simultaneously as formally different phases. The $I(K)$ patterns of the different texture components are measured by adjusting the specimen orientation in an Eulerian cradle.

(2) The dislocation density is found to vary in a wide range between 0.5 and $23 \times 10^{15} \mathrm{~m}^{-2}$ versus both the different textures and the deposition conditions of the thin films.

(3) The Burgers vector population is determined by analyzing the strain component of the line broadening in terms of individual dislocation contrast factors. The matching of the measured and theoretical contrast factors provides the most probable Burgers vectors prevailing in the films. In the $\langle 100\rangle$ - and $\langle 111\rangle$-textured films a considerable fraction of the
Burgers vectors are parallel to the plane of the films. In the $\langle 211\rangle$-textured films, however, the Burgers vectors are populated randomly.

(4) The coherent domain size varies between about 20 and $160 \mathrm{~nm}$ depending on the different textures and the deposition conditions of the thin films. At the same time it is in good correlation with the grain size observed by electron microscopy.

(5) The mechanical strength of the thin films is measured by the Vickers hardness test and is correlated with the combined Taylor and Hall-Petch equation.

(6) The substructure parameters are correlated with the deposition conditions. It is found that the largest current density and the largest amount of additive produce the highest dislocation density and the largest strength value.

(7) The film containing the largest value of dislocation densities, i.e. $23 \times 10^{15} \mathrm{~m}^{-2}$, has been annealed at $673 \mathrm{~K}$ for $30 \mathrm{~min}$. This relatively mild heat treatment reduced the dislocation density by a factor of more than 100 , substantiating the high dislocation density value measured in the as-deposited state of this particular thin film.

KP and HA acknowledge the Danish Research Council for Technology and Production Sciences (grant No. 274-07-0492) for financial support. GC and TU are grateful to the Hungarian National Science Foundation (OTKA Nos. 71594, 67692) for the support of this X-ray peak profile analysis work. The European Union and European Social Fund have provided financial support to this project under grant agreement No. TAMOP 4.2.1./B-09/1/KMR-2010-0003 (to TU).

\section{References}

Akasheh, F., Zbib, H. M., Hirth, J. P., Hoagland, R. G. \& Misra, A. (2007). J. Appl. Phys. 101, 084314.

Alimadadi, H., Bastos, S. A. \& Pantleon, K. (2012). In preparation. Andricacos, P. C., Uzoh, C., Dukovic, J. O., Horkans, J. \& Deligianni, H. (1998). IBM J. Res. Dev. 42, 567-573.

Balogh, L., Ribárik, G. \& Ungár, T. (2006). J. Appl. Phys. 100, 023512.

Borbély, A., Dragomir-Cernatescu, J., Ribárik, G. \& Ungár, T. (2003). J. Appl. Cryst. 36, 160-162.

Cheary, R. W., Dooryhee, E., Lynch, P., Armstrong, N. \& Dligatch, S. (2000). J. Appl. Cryst. 33, 1271-1283.

Clausen, B., Lorentzen, T. \& Leffers, T. (1998). Acta Mater. 46, $3087-$ 3098.

Demkowicz, M. J. \& Hoagland, R. G. (2008). J. Nucl. Mater. 372, 4552.

Erb, U. (2010). Key Eng. Mater. 444, 163-188.

Groma, I. (1998). Phys. Rev. B, 57, 7535-7542.

Hansen, K. \& Pantleon, K. (2008). Scr. Mater. 58, 96-98.

Hansen, N. (2004). Scr. Mater. 51, 801-806.

Hinds, W. C. (1982). Aerosol Technology: Properties, Behaviour and Measurement of Airbone Particles. New York: Wiley.

Krivoglaz, M. A. (1969). Theory of X-ray and Thermal Neutron Scattering by Real Crystals. New York: Plenum Press.

Levay, A., Möbus, G., Vitek, V., Rühle, M. \& Tichy, G. (1999). Acta Mater. 47, 4143-4152.

Lu, L., Chen, X., Huang, X. \& Lu, K. (2009). Science, 30, 607-610.

Moffat, T. P. \& Jossell, D. (2010). Isr. J. Chem. 50, 312-320.

Natter, H. \& Hempelmann, R. (2008). Z. Phys. Chem. 222, 319354.

Pantleon, K. \& Somers, M. A. J. (2006). J. Appl. Phys. 100, 114319. 


\section{research papers}

Pantleon, K. \& Somers, M. A. J. (2010). Mater. Sci. Eng. A, 528, 6571.

Petch, N. J. (1953). J. Iron Steel Inst. London, 173, 25-28.

Ribárik, G., Gubicza, J. \& Ungár, T. (2004). Mater. Sci. Eng. A, 387389, 343-347.

Taylor, G. I. (1934). Proc. R. Soc. London Ser. A, 145, 362-387.

Ungár, T. (2008). Powder Diffr. 23, 125-132.

Ungár, T. \& Tichy, G. (1999). Phys. Status Solidi (a), 147, 425-434.
Ungár, T., Tichy, G., Gubicza, J. \& Hellmig, R. J. (2005). Powder Diffr. 20, 366-375.

Warren, B. E. (1959). Prog. Metal Phys. 8, 147-202.

Weil, R. (1989). Annu. Rev. Mater. Sci. 19, 165-182.

Wilkens, M. (1970). Fundamental Aspects of Dislocation Theory, edited by S. A. Simmons, R. de Wit \& R. Bullough, Vol. II, National Bureau of Standards Special Publication No. 317, p. 1195. Washington, DC: US Government Printing Office. 